Nota 



\title{
La integración de las teorías de la demanda agregada y del circuito monetario en la perspectiva pos-keynesiana de Sergio Cesaratto
}

\author{
Federico Gutiérrez Naranjo $\circledast^{a}$
}

-Introducción -I. Teoría de la demanda agregada y teoría monetaria endógena -II. Integración de la demanda agregada y la teoría endógena, ¿un posible regreso a la ley de Say? -III. Teoría del circuito monetario, y financiación inicial y final de la inversión -IV. Integración teórica propuesta por Sergio Cesaratto -Conclusiones -Referencias.

Primera versión recibida el 14 de abril de 2021; versión final aceptada el 29 de mayo de 2021

\section{Introducción}

Las dos principales críticas que el pensamiento económico neoclásico ha recibido por parte del pensamiento pos-keynesiano son la teoría de la demanda agregada — rechazo de la ley de Say, independencia de la inversión con respecto al ahorro y la tasa de interés, el crecimiento impulsado por la demanda, el súper-multiplicador, etcétera- y la teoría monetaria heterodoxa - rechazo de la tasa de interés natural, teoría endógena del dinero, teoría del circuito monetario, etcétera — 1 .

Estas dos áreas del pensamiento pos-keynesiano a menudo se han desarrollar de manera independiente la una de la otra. En Cesaratto (2016) y Cesaratto y Bucchianico (2020), Sergio Cesaratto propone un marco interpretativo para integrar de manera coherente algunos de los elementos de

a Federico Gutiérrez. Naranjo: estudiante de maestría de la Sorbonne Université y la Università di Roma tre. Dirección electrónica: federicogutierreznaranjo@gmail.com

https://orcid.org/0000-0002-1444-7116

1 Pivetti (2015) expone que Marx y Keynes comparten las tres principales críticas a la comprensión del sistema capitalista por parte de la teoría neoclásica, a saber: la teoría de la demanda agregada, la teoría monetaria de la producción, y parte del análisis de la distribución de la producción. 
la teoría de la demanda agregada y la teoría monetaria heterodoxa. Además, como se muestra más adelante, esta integración permite rechazar una aparente contradicción que puede surgir entre la teoría de la demanda agregada y la teoría del financiamiento de la inversión en el marco de circuito monetario.

El objetivo de este texto es presentar de la forma más clara y esquemática posible la propuesta teórica de Sergio Cesaratto, con el fin de contribuir a la difusión de las teorías pos-keynesianas ${ }^{2}$ en español teniendo en cuenta la importancia analítica que siguen teniendo para la comprensión del funcionamiento del capitalismo. Para esto se toma como base los artículos de Cesaratto (2016) y Cesaratto y Bucchianico (2020), y la bibliografía esencial utilizada en ellos.

El presente texto está dividido en cuatro partes interconectadas: la primera parte expone algunos elementos básicos de la teoría de la demanda agregada y de la teoría monetaria endógena; la segunda, integra estas dos teorías y expone la posible contradicción y paradoja que puede surgir de allí la tercera parte muestra los elementos básicos de la teoría del circuito monetario, y de la financiación inicial y final de la inversión; la cuarta parte desarrolla la propuesta teórica de Sergio Cesaratto que permite solventar la aparente contradicción ya mencionada; y por último se cierra el texto con algunos comentarios finales.

\section{Teoría de la demanda agregada y teoría del dinero endógeno}

\section{A. Teoría de la demanda agregada}

Para los propósitos del texto es necesario abordar de manera sumaria dos elementos que se desarrollan dentro de las teorías de la demanda agregada: el multiplicador y el súper-multiplicador.

La teoría de la demanda agregada postulada por Keynes rechaza varios de los pilares teóricos de la teoría neoclásica como la Ley de Say — la oferta crea su propia demanda de equilibrio a través de la flexibilidad salarial y cambios

2 No debe confundirse con la síntesis neoclásica de corte Keynesiana ni con el neo-keynesianismo. La teoría pos-keynesiana es representada por autores como Joan Robinson, Michal Kalecki, Pierangelo Garegnani, Massimo Pivetti, Marc Lavoie, Engelbert Stockhammer, entre otros. 
Gutiérrez Naranjo: La integración de las teorías de la demanda agregada y del circuito...

en la tasa de interés ${ }^{3}$ - la teoría de la producción por el lado de la oferta - esto es, la producción es determinada por la maximización de los factores de producción con base en la relativa escasez de estos y las preferencias de los consumidores-, y la dependencia de la inversión con respecto a los ahorros y la tasa de interés. Por el contrario, la teoría de la demanda agregada invierte estas relaciones al afirmar que no existen mecanismos autónomos que conduzcan a la economía al pleno uso de los factores productivos, que la producción es determinada por la demanda, y que las inversiones son cuasiindependientes de los ahorros y la tasa de interés. Es más, la inversión crea su propio ahorro a través de cambios en la producción por medio del efecto multiplicador, como se muestra en la Figura 1.

Figura 1. Efecto del multiplicador de la inversión

\begin{tabular}{|c|c|c|c|c|c|c|c|}
\hline \multicolumn{8}{|c|}{ Investment multiplier (received view) } \\
\hline Periods & & $\Delta \mathrm{Y}$ & $\Delta \mathrm{C}$ & \multicolumn{2}{|c|}{$\Delta S \quad \Delta$ deposits } & $\Delta \mathrm{H}$ & $\Delta \mathrm{E}$ \\
\hline 1 & 100 & \multicolumn{2}{|c|}{$100 \because:$} & & 100 (loan) & & \\
\hline 2 & & \multicolumn{2}{|c|}{$80 \div 80$} & $\rightarrow 20.0$ & 82.0 & 2.0 & 18.0 \\
\hline 3 & & \multicolumn{2}{|c|}{$64 \longleftarrow-64$} & $\rightarrow 16.0$ & 67.6 & 1.6 & 14.4 \\
\hline 4 & & 51.2 & 51.2 & 12.8 & 56.1 & 1.3 & 11.5 \\
\hline 5 & & 40.9 & 40.9 & $10.2 \rightarrow$ & 46.9 & 1.0 & 9.2 \\
\hline$\ldots$ & & $\ldots$ & $\ldots$ & $\ldots$ & & $\ldots$ & $\ldots$ \\
\hline Final & & 500 & 400 & 100 & 10 & 10 & 90 \\
\hline
\end{tabular}

Fuente: Cesaratto y Bucchianico (2020).

La Figura 1 ejemplifica la forma en la que una inversión autónoma inicial genera un proceso multiplicador en la economía de forma tal que la producción aumenta, y este aumento en la producción es el que permite obtener unos ahorros iguales a la inversión inicial. La inversión inicial es de 100 unidades monetarias, la propensión a consumir es de 0.8 y la propensión a

3 "En la base de la interpretación neoclásica de la ley de Say está su teoría de la distribución del ingreso: la concepción neoclásica del salario como la variable que iguala la demanda y la oferta del trabajo, y de la tasa de interés o ganancia — la remuneración del capital— como la variable que iguala la demanda y la oferta de ahorro, es decir, la que asegura que toda la parte del producto no absorbida por el gasto en consumo sea absorbida por el gasto en inversión". (Pivetti, 2002, p.5) [traducción propia]. 
ahorrar es de 0.2. La inversión inicial genera una serie de consumos y ahorros que se prolongan en el tiempo y que van disminuyendo. Al final, el efecto multiplicador genera 100 unidades monetarias de ahorro y 400 de consumo, para un total de 500 unidades monetarias adicionales en la producción.

Frente a este proceso es importante realizar dos comentarios. El primero, es que es analíticamente válido cuando la economía no se encuentra en su máxima utilización de los factores productivos, premisa que se corresponde con el funcionamiento normal de las economías - por el contrario, es difícil corroborar empíricamente la premisa neoclásica de la máxima utilización de los factores productivos-. El segundo, es recalcar que la distinción entre cambios en liquidez $(\Delta H)$ y cambios en la compra de deuda $(\Delta E)$, influenciada por el trabajo de Dalziel (1996), es esencial para una mejor comprensión del proceso. Esto es, los ahorros pueden estar en forma de dinero líquido o en forma de bonos. Sin embargo, al final del proceso, la suma de la posesión líquida de dinero más compra de deuda es igual al monto de la inversión inicial.

Partiendo de la teoría de la demanda agregada los pos-keynesianos también han sugerido una teoría del crecimiento económico conocida como el súper-multiplicador. A la identidad macroeconómica básica ${ }^{4} Y=C+$ $I+G+X-M$, se incluye de manera explícita el funcionamiento del multiplicador, el acelerador de la inversión ${ }^{5}$, y se divide analíticamente los componentes autónomos e inducidos de la demanda. Los componentes autónomos de la demanda son aquellos que no dependen del ingreso presente, como las exportaciones, alguna parte de la inversión y el consumo, y el gasto del gobierno. Los componentes inducidos de la demanda son aquellos que dependen del ingreso, como una parte de la inversión y la mayor parte del consumo. La ecuación resultante es la siguiente (Girardi \& Pariboni, 2016):

$4 \quad Y$ es la producción total, $C$ es el consumo de los hogares, $I$ es la inversión, $G$ es el gasto del gobierno, $X$ son las exportaciones y $M$ son las importaciones.

5 El multiplicador keynesiano es el efecto que genera un aumento en el consumo sobre la demanda agregada, mientras que el acelerador es el efecto que genera un aumento en la demanda agregada sobre la inversión. Estos dos procesos se suelen potenciar mutuamente. Para una explicación de la integración de ambos principios se recomienda Samuelson (1939). 
Gutiérrez Naranjo: La integración de las teorías de la demanda agregada y del circuito...

$$
Y=\frac{C o+G+X}{s+m-h}=\frac{Z}{s+m-h},
$$

donde $s, m$ y $h$ son parámetros y $Z$ reúne los componentes autónomos de la demanda - esto es, el consumo autónomo, el gasto del gobierno y las exportaciones 6 - . Lo importante para los propósitos de este trabajo es la separación analítica de los elementos autónomos e inducidos de la demanda. En particular, el consumo autónomo que, al no estar determinado por los ingresos, es financiado por creación de dinero, y por esta vía se une con la teoría endógena del dinero que se analiza a continuación: "Es característico del gasto autónomo no ser financiado por los ingresos, como sucede en el caso del gasto inducido; debe ser, entonces, financiado por creación crediticia” (Cesaratto, 2016, p. 5) [traducción propia].

\section{B. Teoría endógena del dinero}

Parte de la teoría neoclásica supone que la oferta de dinero es exógena al sistema económico y que esta depende las decisiones de emisión monetaria del Banco Central ${ }^{7}$. La teoría monetaria endógena, por el contrario, argumenta que la creación de dinero se determina dentro del sistema mismo (de allí lo endógeno) a través del sistema financiero.

Para la teoría del dinero endógeno, el banco central establece la tasa de interés y, a partir de esta, los agentes económicos demandan créditos a los bancos comerciales. La efectiva creación monetaria depende de la demanda efectiva de créditos por parte de los agentes y no de una decisión autónoma del banco central ${ }^{8}$.

6 Para una explicación de la teoría del súper-multiplicador se recomienda el artículo de Serrano y Freitas (2015). Para una corroboración empírica de la teoría del súper-multiplicador, el artículo de Girardi y Pariboni (2016).

7 Esto se puede evidenciar con mayor claridad teórica en el modelo IS-LM de corte neoclásico, en el cual la oferta de dinero dentro de la curva LM es vertical y determinada por el Banco Central. Por el contrario, una concepción del dinero endógena haría la curva de oferta monetaria horizontal o casi-horizontal.

8 Para profundizar sobre la teoría endógena del dinero se recomienda la primera sección del libro editado por Piégay y Rochon (2006). 
Según esta teoría no existen límites a la creación monetaria —o los límites son muy altos - por parte de los bancos comerciales, dado que la Banca Central acomoda sus depósitos y reservas para satisfacer las demandas de moneda a una determinada tasa de interés. Esta lógica se acomoda más al real funcionamiento de la emisión monetaria y el funcionamiento del sistema financiera, al punto que McLeay et al. (2014) exponen explícitamente en la revista del Banco Central de Inglaterra esta teoría como el modelo de funcionamiento del sistema:

Otra mal entendido es que el banco central determina la cantidad de préstamos y depósitos en la economía controlando la cantidad de dinero por parte del banco central [...] (en realidad), los bancos deciden cuándo desean prestar dependiendo de las oportunidades de ganancias disponibles — las cuales dependen de la tasa de interés fijada por el Banco de Inglaterra- Es entonces estas decisiones de préstamos las que determinan cuántos depósitos son creados por el sistema bancario. La cantidad de depósitos bancarios, a su vez, influencia cuánto dinero desea la banca central mantener como reservas (para satisfacer la liquidez del público, realizar pagos a otros bancos, o igualar la liquidez requerida legalmente) que son luego suministrados a demanda por el banco central. (p. 15) [traducción propia].

\section{Integración de la demanda agregada y la teoría endógena, ¿un posible regreso a la ley de Say?}

Es posible integrar la teoría de la demanda agregada —en particular, la importancia que tiene el crédito en dinamizar los componentes autónomos de la demanda - y la teoría del dinero endógeno, así: la creación de dinero endógena por parte de los bancos permite financiar el consumo o inversión de manera independiente de los ingresos o ahorros previos, y reafirma la demanda como la causa de la producción y el crecimiento económico. A esta situación Cesaratto (2016) la denomina como la "visión heredada" de la teoría pos-keynesiana. 
Gutiérrez Naranjo: La integración de las teorías de la demanda agregada y del circuito...

Sin embargo, hay ciertas ocasiones en que esto puede ser problemático analíticamente. Por ejemplo, cuando la producción precede a la demanda efectiva y al pago de esta: "En muchos casos prácticos, la producción es generada a partir de las expectativas de la demanda efectiva de bienes estandarizados, o por órdenes de compra, por ejemplo, en complejos y/o personalizados pedidos industriales, o bienes de lujo" (Cesaratto, 2016, p. 7) [traducción propia]. En estos casos la firma debe iniciar la producción sin la demanda ni el pago, y la pregunta por cómo se financia surge de nuevo. Sin embargo, es importante resaltar que en este caso la pregunta ya no es por cómo se financia el consumo sino la producción. Es posible responder que la producción también es financiada por la creación de dinero endógeno por medio de los bancos comerciales:

Esto asignaría un rol adicional a los bancos —llamémoslo financiación inicial - en financiar la producción durante la gestación del periodo productivo, esto es, durante el periodo en el que las expectativas de compra o la producción se realiza, y el periodo en que se entrega y se realiza el pago final del bien (Cesaratto, 2016, p. 8) [traducción propia].

Pero si esto es así, surge inevitablemente la siguiente pregunta:

¿Acaso el rol de la creación endógena de dinero en financiar la oferta con base en las expectativas contradice el principio de la demanda efectiva? Esto es, ¿no se corre el riesgo de, implícitamente, restaurar la ley de Say en la que la oferta crea su propia demanda? (Cesaratto, 2016, p. 8) [traducción propia].

Esto es, si la producción es financiada por las empresas de manera independiente de la demanda efectiva y del pago de esta, el inicio de la producción depende de las decisiones de las empresas y por esta vía una restauración de la ley de Say en la cual la producción crea su propia demanda? — es decir, un regreso a la visión de la producción del lado de la oferta—.

9 No es la primera vez que surgen aparentes incompatibilidades entre el principio de la demanda agregada y la financiación por medio de dinero endógeno. En Dalziel (1996) se analiza —y 
Esta paradoja del posible regreso de la ley de Say a partir de las teorías pos-keynesianas es sólo aparente, en realidad no sucede tal cosa. La respuesta analítica que propone Sergio Cesaratto permite aclarar esta confusión e integrar parte de la teoría de la demanda agregada con la teoría del circuito monetario. Pero antes de abordar esto, es necesario hacer un breve comentario en la próxima sección sobre la teoría del circuito monetario, y la teoría de la financiación inicial y final.

\section{Teoría del circuito monetario, y financiación inicial y final de la inversión}

\section{A. La teoría del circuito monetario}

La teoría del circuito monetario describe el funcionamiento circular de la creación, uso y destrucción del dinero dentro del sistema productivo. Guarda una relación estrecha con la economía de la producción monetaria postulada por Marx en la forma $M-C-M$ ', y hace parte de las llamadas teorías monetarias heterodoxas al reconocer la importancia del dinero en la producción y no meramente — como sostiene la escuela neoclásica- un medio de intercambio. Además, su enunciación conlleva implícitamente la teoría del dinero endógena ya mencionada. Hay diversos modelos del circuito monetario, pero su postulación básica del flujo monetario se puede observar en la Figura 2.

El modelo básico cuenta con tres agentes: banco comercial, firma y hogares. A continuación, se explica el sentido del flujo monetario:

1. Para empezar el proceso productivo, la empresa pide prestado al banco comercial el dinero suficiente para cubrir los gastos iniciales (pago de salarios).

se resuelve- un problema similar conocido como el debate Cottrell-Moore: "Si estas dos teorías no son compatibles, como Moore argumenta, esto representa un problema serio en la coherencia interna del proyecto pos-keynesiano. El debate Cottrell-Moore se da en el análisis de las consecuencias de un aumento de la inversión financiada por creación de dinero de manera endógena” (p. 312) [traducción propia]. 
Gutiérrez Naranjo: La integración de las teorías de la demanda agregada y del circuito...

2. El banco crea moneda - creación endógena del dinero-y de esta manera se abre el circuito monetario. Esta creación monetaria debe ser entendida como un registro contable en del banco.

3. Al recibir el dinero por parte del banco, la empresa puede entonces pagar los salarios y gastos iniciales, y empezar la producción.

4. La producción de los bienes termina y puede suceder dos cosas: 4.1) los salarios son gastados en su totalidad en la compra de la mercancía producida por la empresa. En este caso la empresa recoge el monto total de los salarios y el saldo suficiente para pagar la deuda con el banco; o 4.2) los salarios no son gastados en su totalidad en la compra de los bienes y se genera un ahorro por parte de los asalariados. En este caso, la empresa recoge parte de los salarios, pero para recuperar el resto debe contraer deuda (bonos) con los trabajadores por el valor de sus ahorros.

5. Sea la situación 4.1 o 4.2, la empresa recupera el saldo total de la deuda inicialmente contraída y la salda. El banco comercial elimina la deuda de sus contabilidades y el dinero es "destruido". A este proceso se le denomina el flujo y reflujo del dinero.

Figura 2. Circuito monetario básico

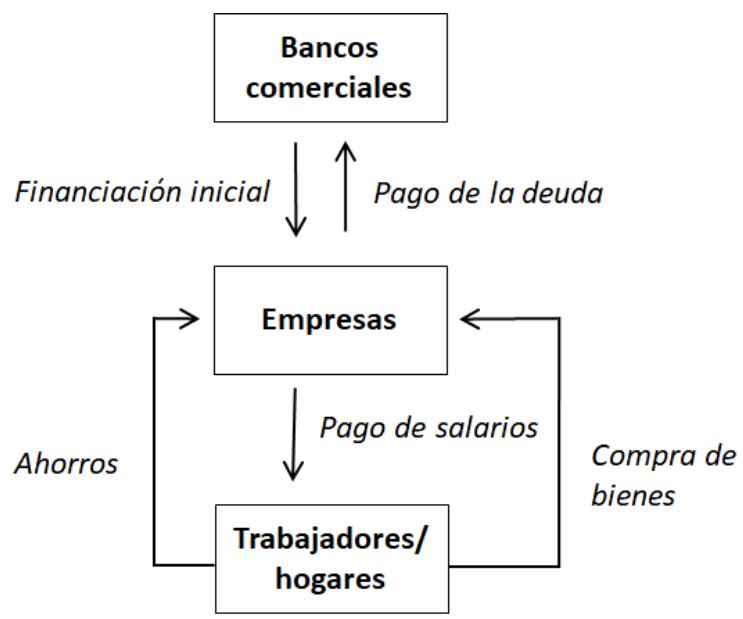

Fuente: Realfonzo (2006). 
El proceso apenas descrito corresponde al modelo básico del circuito monetario, y es suficiente para los objetivos del presente texto ${ }^{10}$. Sin embargo, existen circuitos más completos y complejos donde se incluye a la banca central, el gobierno y la economía externa.

\section{B. Financiación inicial y final de la inversión}

Como se enunció en la sección II, puede haber dos momentos claros y distinguibles de financiación por parte de los bancos comerciales: la financiación inicial a la producción, y la financiación final a la demanda en la que el inversor o consumidor paga los bienes. Davidson (1986) explica la lógica que subyace a este doble movimiento:

El sistema bancario cumple con la función de crear financiación adicional de corto plazo cuando los empresarios desean aumentar el nivel de flujo de la inversión real. Esta financiación creada por la banca (que no utiliza recursos previos) debe ser distinguida del rol de la financiación de largo plazo en la que se requiere que el público renuncie a una determinada cantidad de liquidez igual a los ahorros reales (ex. no reclamo de ingresos obtenidos) en el proceso de financiamiento de la inversión (p. 101) [traducción propia].

En este sentido, tanto la financiación inicial de la producción como el consumo autónomo son sustentadas en un primer momento a través de la creación endógena de dinero, pero al final la inversión es financiada por compra de deuda sustentada en los ahorros o depósitos generados por el circuito y el multiplicador de la inversión inicial.

Partiendo de este análisis, y trayendo elementos de Dalziel (1996) ${ }^{11}$, Sergio Cesaratto propone un esquema integrativo de algunos elementos del principio de la demanda afectiva y la teoría del circuito monetario, que permite aclarar la paradoja enunciada en la sección II.

10 Para profundizar sobre la teoría del circuito monetario se recomiendan los artículos de Realfonzo (2006) y Claude Gnos (2006), o el libro de Graziani (2003).

11 En especial, lo referente a la diferenciación de flujos financieros y flujos reales dentro del proceso del multiplicador keynesiano. 
Gutiérrez Naranjo: La integración de las teorías de la demanda agregada y del circuito...

\section{Integración teórica propuesta por Sergio Cesaratto}

Es posible integrar los elementos analizados hasta el momento en un circuito monetario y de la producción coherente que, además, permitan resolver la aparente contradicción enunciada la sección II. Para esto se utiliza un ejemplo similar al utilizado por Cesaratto (2016), a través de momentos secuenciales los cuales están representados en la Figura 3 y explicados a continuación. El caso incluye cuatro agentes: una firma productora, un comprador, un banco comercial y un banco de inversión. El ejemplo muestra los flujos financieros y reales que se generan con la compra de un avión por parte del comprador a la firma productora.

Figura 3. Integración propuesta por Sergio Cesaratto

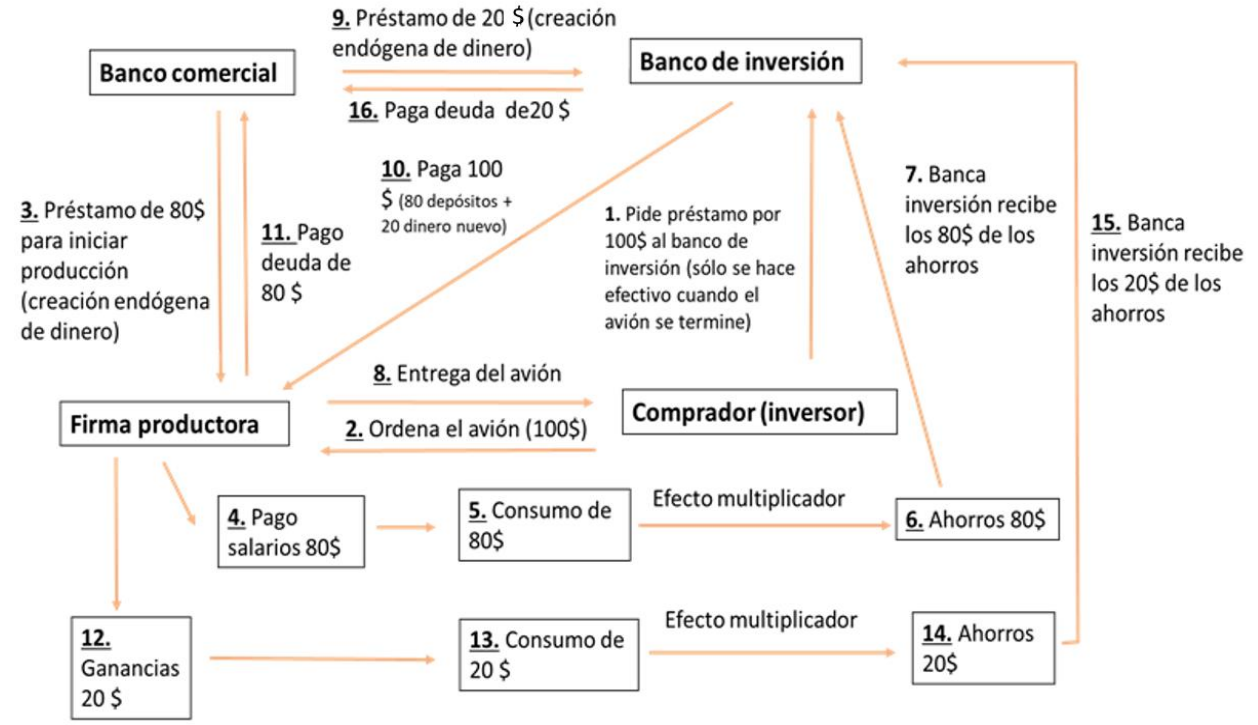

Fuente: elaboración propia.

Momentos 1 y 2: una empresa desea comprar mediante endeudamiento un avión cuyo valor son $\$ 100$. Antes de realizar la orden asegura la disponibilidad del dinero mediante un contrato con un banco de inversión en el cual se comprometen a que, llegado el momento de la entrega, el banco 
le genera un préstamo por los $\$ 100$. Una vez asegurado el préstamo la empresa encarga el avión.

Momentos 3 y 4: la firma productora del avión debe iniciar la producción. Para esto necesita de dinero para pagar los gastos iniciales — salarios, que equivalen a $\$ 80$ - y por lo tanto genera un préstamo con un banco comercial. El banco comercial financia los $\$ 80$ mediante creación endógena de dinero.

Momentos 5, 6 y 7: el pago de los $\$ 80$ en salarios empieza a circular en la economía y, a través del multiplicador genera un monto igual de ahorros (ver Figura 1) que, luego, irán a parar en la cuenta de depósitos bancarios o compra de títulos. En cualquier caso, este saldo de dinero es canalizado a través de la banca de inversión.

Momentos 8, 9 y 10: la firma productora termina el avión y se lo entrega a la empresa compradora. Esta debe pagar el avión por lo que hace efectivo el contrato con el banco de inversión para el desembolso de los $\$ 100$. El banco de inversión tiene $\$ 80$ de los ahorros generados en los pasos anteriores, y para completar los $\$ 20$ restantes, realiza un préstamo con el banco comercial por \$20 que lo financia a través de creación endógena de dinero.

Momentos 11 y 12: la firma productora recibe el pago de los $\$ 100$, y podrá saldar la deuda de $\$ 80$ con el banco comercial (acá se cierra el "primer" circuito y el dinero es “eliminado"). Los $\$ 20$ restantes son la ganancia de la firma ${ }^{12}$.

Momentos 13, 14, 15 y 16: las ganancias generadas inician a circular en la economía y a gastarse en el mercado y, a través del efecto multiplicador (ver Figura 1) generan ahorros iguales a $\$ 20$. Estos ahorros son canalizados por medio de la banca de inversión que puede ahora saldar la deuda de $\$ 20 \$$ con la banca comercial. El resultado final es que la banca comercial efectivamente financió los $\$ 100$ por medio de creación endógena de dinero,

12 Una de las cuestiones problemáticas con la teoría del circuito monetario es la explicación de las ganancias. Sin embargo, esta aproximación teórica provee una descripción del proceso de formación de las ganancias: "en línea con Kalecki, los capitalistas ganan lo que gastan, y el bien conocido problema de los circuitos monetarios de cómo se generan las ganancias no aparece acá” (Cesaratto \& Bucchianico, 2020, p. 12) [traducción propia]. 
Gutiérrez Naranjo: La integración de las teorías de la demanda agregada y del circuito...

pero la financiación de la inversión estuvo soportada, al final, por los ahorros generados mediante el proceso productivo.

\section{Conclusiones}

Retomando, en la sección II se planteó la paradoja de regresar a la ley de Say — y por lo tanto a la teoría de la oferta neoclásica - partiendo precisamente de las teorías pos-keynesianas. La respuesta es que tal paradoja no existe, y por el contrario ambas teorías se refuerzan. Es posible complementar esto con las siguientes precisiones.

Como se evidencia en el momento 1, las decisiones de inversión son independientes de los ahorros, en contraposición a la visión neoclásica que considera la inversión como el resultado del equilibro entre los ahorros previos y la tasa de interés.

Del momento 3, se tiene que la decisión de producción sigue estando determinada por el principio de la demanda efectiva, aun cuando esta se presente bajo expectativas de demanda efectiva. Esto, en contraposición a la postura neoclásica en la que las decisiones de producción dependen de la maximización de los factores de producción disponibles.

Los momentos 5-6 y 13-14, evidencian que la inversión genera sus propios ahorros reales mediante el aumento del ingreso a través del multiplicador keynesiano. La inversión real es financiada con liquidez real que generó esa inversión. Esto, en contraposición a la teoría neoclásica en la cual son los ahorros previos los que sustentan y financian las inversiones futuras.

Es importante aclarar que el proceso descrito en la sección IV no se corresponde con todos los procesos y flujos económicos - por ejemplo, no todos los pagos de salarios son previos a la producción-. Sin embargo, su importancia radica en las posibilidades analíticas que permite hacer sobre el sistema capitalista moderno. Siguiendo este argumento, vale la pena resaltar que en el artículo Cesaratto y Bucchianico (2020) se presenta el proceso descrito en la sección IV, pero desagregado de manera analítica para cada uno de los componentes del gasto autónomo (para el consumo autónomo, el gasto del gobierno y las exportaciones e importaciones en una economía abierta). 
Este texto intentó mostrar de la forma más clara posible una forma de integrar la teoría de la demanda agregada y la teoría del circuito monetario sugerida por el economista Sergio Cesaratto. Pero en el desarrollo del argumento se pasa — aunque de manera sumaria - por varias de las ramas en las que es posible evidenciar cómo las teorías pos-keynesianas difieren de las teorías neoclásicas. Sin embargo, a pesar de existir distintos marcos interpretativos del actual sistema económico, la enseñanza de la economía está dominada de manera casi que exclusiva por la escuela neoclásica. Es por esto que este texto también debe ser visto como un intento por difundir las teorías pos-keynesianas en español, y de esta manera contribuir a enriquecer el debate y el análisis de los fenómenos económicos.

\section{Referencias}

Cesaratto, S. (2016). Initial and Final Finance in the Monetary Circuit and the Theory of Effective Demand. Metroeconomica, 68(2), 228-258. https: //doi.org/10.1111/meca.12132

Cesaratto, S., \& Bucchianico, S. d. (2020). Endogenous Money and the Theory of Long Period Effective Demand. Bulletin of policital economy 14(1), 1-38. https://serialsjournals.com/abstract/38226_1-cewsaratt o_di_bucchianico.pdf

Dalziel, P. (1996). The Keynesian Multiplier, Liquidity Preference, and Endogenous Money. Journal of Post Keynesian Economics, 18(3), 311-331. https://doi.org/10.1080/01603477.1996.11490075

Davidson, P. (1986). Finance, Funding, Saving, and Investment. Journal of Post Keynesian Economics, 9(1), 102-110. https://www.jstor.org/stable 14537993

Freitas, F., \& Serrano, F. (2015). Growth Rate and Level Effects, the Stability of the Adjustment of Capacity to Demand and the Sraffian Supermultiplier. Review of Political Economy, 27(3), 258-281. https: //doi.org/10.1080/09538259.2015.1067360 
Girardi, D., \& Paribon, R. (2016). Long-run Effective Demand in the US Economy: An Empirical Test of the Sraffian Supermultiplier Mode. Review of Political Economy, 28(4), 523-544. https://doi.org/10.108 $0 / 09538259.2016 .1209893$

Gnos, C. (2006). French circuit theory. En M. Sawyer, \& P. Arestis, A Handbook of Alternative Monetary Economics (pp. 87-104). Edward Elgar.

Graziani, A. (2003). The monetary theory of production. Cambridge University Press. Edward Elgar.

McLeay, M., Radia, A., \& Thomas, R. (2014). Money creation in the modern economy [Quarterly Bulletin of the Bank of England, Q1]. https: //www.bankofengland.co.uk/-/media/boe/files/quarterly-bulletin /2014/money-creation-in-the-modern-economy.pdf?la=en\&hash=9A 8788FD44A62D8BB927123544205CE476E01654

Piégay, P., \& Rochon, L. (2006). Teorías monetarias poskeynesianas. AKAL editorial.

Pivetti, M. (2002). Economia Politica. Laterza.Pivetti, M. (2015). Marx and the Development of Critical Political Economy. Review of Political Economy, 27(2), 134-153. https://doi.org/10.1080/09538259.201 5.1010706

Realfonzo, R. (2006). The Italian Circuitist Approach. En M. Sawyer, \& P. Arestis, A Handbook of Alternative Monetary Economics (pp. 105121). Edward Elgar.

Samuelson, P. (1939). Interactions between the Multiplier Analysis and the Principle of Acceleration. The review of Economics and Statistics, 21 (2). https://doi.org/10.2307/1927758

Cómo citar / How to cite this item:

Gutiérrez Naranjo F. (2022). La integración de las teorías de la demanda agregada y del circuito monetario en la perspectiva pos-keynesiana de Sergio Cesaratto. Lecturas de Economía, 96, 345-360. https://doi.org/10.17533/udea.le.n96a345977 